\title{
Outcome measures of risk and recovery in Broadmoor High Secure Forensic Hospital: stratification of care pathways and moves to medium secure hospitals
}

Samantha McCullough, Carolyn Stanley, Helen Smith, Molly Scott, Minesh Karia, Benignus Ndubuisi, Callum C. Ross, Rob Bates and Mary Davoren

\section{Background}

Placements within high secure forensic hospitals consist of wards providing various different levels of relational security. They should form a coherent pathway through secure care, based on individual patient risks and needs. Moves to less secure wards within high secure forensic hospitals and moves on to lower secure hospital settings have rarely been systematically studied.

\section{Aims}

The aim of this study was to ascertain if placements within Broadmoor High Secure Hospital and moves from Broadmoor to medium secure hospitals corresponded to measures of violence risk, programme completion and recovery.

\section{Method}

A 13-month prospective cohort study was completed. Patients $(n=142)$ were rated at baseline for violence risk (Historical, Clinical and Risk - 20), therapeutic programme completion and recovery (DUNDRUM tool) and overall functioning (Global Assessment of Functioning). Placements on the care pathway and moves on to medium secure hospitals were observed.

\section{Results}

Placements on the care pathway within the high secure hospital were associated with dynamic violence risk ( $F=16.324$, $P<0.001)$, therapeutic programme completion $(F=4.167$, $P=0.003)$, recovery $(F=2.440, P=0.050)$ with better scores on these measures being found in the rehabilitation wards and the poorest scores on the highest levels of dependency. Moves to medium secure hospitals were associated with better scores on dynamic risk of violence $(F=33.199, P<0.001)$, therapeutic programme completion $(F=9.237 P<0.001)$, recovery $(F=6.863$, $P=0.001)$.

\section{Conclusions}

Placements within Broadmoor Hospital formed a coherent pathway through high secure care. Moves to less secure places were influenced by more than reduction in violence risk. Therapeutic programme completion and recovery in a broad sense were also important.

\section{Keywords}

Forensic mental health services; high security hospitals; outcome measures; violence risk; recovery.

\section{Copyright and usage}

(C) The Author(s) 2020. This is an Open Access article, distributed under the terms of the Creative Commons AttributionNonCommercial-NoDerivatives licence (http://creativecommons.org/licenses/by-nc-nd/4.0/), which permits non-commercial re-use, distribution, and reproduction in any medium, provided the original work is unaltered and is properly cited. The written permission of Cambridge University Press must be obtained for commercial re-use or in order to create a derivative work.

\section{Background}

Secure forensic mental health services have a dual role, to treat mental illness and reduce violent recidivism. ${ }^{1}$ NHS England provides secure forensic mental healthcare at various different levels of therapeutic security. ${ }^{2}$ However, the appropriate use of placements on secure care pathways and progression along secure care pathways has often evolved organically from clinical practice and has rarely been systematically studied, particularly at high secure levels. ${ }^{3,4}$

High secure forensic mental health services provide care and treatment to mentally disordered offenders who pose the highest risk to the public and in England account for just over 700 beds across three hospitals. ${ }^{5,6}$ By comparison, medium secure beds in England account for 3500 beds across 60 units. ${ }^{5}$ Secure forensic mental health services are low-volume high-cost services and account for $10 \%$ of the mental health budget in NHS England. They are therefore subject to significant scrutiny and critique.

In the Republic of Ireland, high, medium and low secure forensic beds are located on one hospital campus, with significantly higher levels of staffing and therefore higher levels of relational security on the high secure area of the hospital service. ${ }^{8}$ Belgium developed its first high security forensic hospital as recently as
2014 in response to much critique from the European Court of Human Rights regarding the need to provide secure care for mentally disordered offenders. ${ }^{9}$ Similarly in Australia, only a small number of secure forensic beds are provided at high secure level, for example the state of New South Wales has one high secure unit, alongside three medium secure, two low secure and two open forensic units. ${ }^{10}$ Internationally, high secure beds are a scarce and expensive resource. The aim of these high security units is to provide therapeutic security, which encompasses physical and procedural security similar to custodial settings, combined with relational security that is based on good clinical care and staff knowledge of the patient group. ${ }^{1}$

\section{Implementing the 'least restrictive option'}

Provision of mental healthcare in the least restrictive setting appropriate to safely manage the patients' risks and needs is a core principle of mental health law in many jurisdictions. ${ }^{11}$ It is central to the mental health code of practice in England and Wales. ${ }^{12}$ Implementing the 'least restrictive option' for patient placements requires balancing risks and freedoms, rights and responsibilities. Increasingly secure forensic mental health settings are expected 
to reduce restrictive practices, but at the same time eliminate in-patient violence or violence towards staff. This is a constant challenge. Although most forensic mental health services strive to implement the principle of caring for patients in the least restrictive setting, demonstrating that this is taking place is often very challenging to do.

\section{Stratification of care pathways}

Within secure forensic hospitals, the care pathway is typically stratified according to risk, with higher staff to patient ratio's present on wards that accommodate individuals with higher levels of symptoms and higher risks and lower staff numbers on rehabilitation wards. ${ }^{1,8}$ The aim of this system of stratification of the care pathways within secure units is to allow patients graded access to increasing freedoms and increasing personal responsibility. Placement on therapeutically secure care pathways should correspond to risk and need for individual patients and should change over time as patients recover. This system should facilitate patients being placed in the least restrictive hospital setting appropriate to meet their individual risks and needs, but also within those secure hospitals to be placed on the least restrictive unit possible.

Placements within hospitals can significantly affect the freedoms of individual patients, however, it does not always follow that a patient placed at a higher level of security will experience more restrictions. For example, a patient that requires repeated seclusions in a medium secure hospital may in fact have more day-to-day freedom within a high security hospital where the higher relational, procedural and physical security may reduce that individuals need for seclusion. There is currently limited research examining the area of care pathways within secure settings, and little evidence base for decision-making regarding moves between therapeutically secure units, despite this being a key outcome for patients, their families and carers as well as hospitals and health services.

\section{Assessing risk and needs}

The Historical, Clinical and Risk - 20 (HCR-20) violence risk assessment is a widely validated and accepted tool for use in secure forensic mental health settings. ${ }^{13}$ This structured professional judgement (SPJ) instrument is utilised in practice in the majority of secure forensic mental health settings in the UK, the European Union, Canada and Australia. ${ }^{14-18}$ The 20 items rated in HCR-20 are statistically associated with future violence and the clinician retains the final judgement as per the SPJ methodology. ${ }^{13,19}$ It was HCR-20-version 3 (HCR-2-V3) that was used in this study. ${ }^{13}$

The DUNDRUM tool is a needs assessment, not a risk assessment. ${ }^{20}$ It is also an SPJ instrument following a similar rating style as seen in the HCR-20. ${ }^{19}$ The DUNDRUM tool consists of five instruments, the first two, DUNDRUM-triage security and triage urgency are designed to support clinicians managing a waiting list for forensic psychiatric hospital settings. ${ }^{20}$ They rate the level of security a patient should be admitted to and the urgency of need for admission; has good psychometric properties and has been internationally validated. ${ }^{21-27}$ The DUNDRUM-3 programme completion and DUNDRUM-4 recovery scales were used in this study. These tools rate the successful completion of therapeutic programmes by the patients, and rate the patient's overall recovery in a holistic manner, respectively. ${ }^{10,28-32}$

These two tools work together to ascertain the patient's readiness to move to less secure places, for example, from a high secure hospital to a medium secure hospital. The tools are rated ' 4 ' to ' 0 ' in seven domains of programme completion and recovery, with each score linked to a series of definitions. The scores are calibrated in units of meaningful change, for example, when a patient moves from ' 4 ' to ' 3 ' on most items, they would likely be ready to move down one level of therapeutic security. The final tool of the DUNDRUM tool is the self-rated DUNDRUM tool, which is a mirror image of the DUNDRUM-3 and DUNDRUM-4 scales and is designed to support a patient giving their own view on readiness to move to less secure places. ${ }^{33}$ Both the DUNDRUM tool and HCR-20 tool are SPJ instruments, therefore they guide but do not bind, and the final decision always rests with the treating clinician. The DUNDRUM tool has been shown to have utility as an outcome measure in medium and low secure forensic hospital settings internationally, however, this is the first study using the programme completion and recovery measures of the DUNDRUM tool in a high secure forensic hospital. ${ }^{31,32,34,35}$

The Global Assessment of Functioning (GAF) was designed as a tool to assess patients overall functioning as part of DSM-IV. ${ }^{36}$ It is widely used, quickly administered and has very good psychometric properties.

\section{Objectives}

The aim of this study was to ascertain if patient placements within the high security hospital at Broadmoor corresponded to measures of risk, need and recovery. We also aimed to ascertain if grounds access within Broadmoor High Secure Hospital and approval for step-down trial leaves to medium secure hospitals were associated with structured measures of violence risk, recovery in a broad sense, therapeutic programme completion and overall functioning.

\section{Method}

\section{Study design}

This is a 13-month prospective study of a cohort of patients in a high security forensic mental health setting. A 13-month period was chosen as a result of the availability of the research team and also as all in-patients in the hospital have a formal review of progress once every 6 months as standard and therefore all would have at least two case reviews during the follow-up period.

\section{Setting}

Broadmoor Hospital is England's oldest high security hospital, offering in-patient care and treatment to individuals with mental disorder who are considered to pose a grave and immediate risk to the public. Of note, England is a jurisdiction where compulsory treatment is not permitted in prison and therefore individuals requiring compulsory treatment for mental disorder must be admitted to a hospital setting for such treatment to take place. Very high security needs have been demonstrated among patients admitted to Broadmoor Hospital, both in comparison with medium secure admissions in the UK and medium and high secure admissions in other jurisdictions. ${ }^{6}$

All in-patients are men, over 18 years and all detained under the Mental Health Act 1983. Care is offered along two therapeutic pathways, a mental illness pathway for individuals with primary Axis I mental disorders and a personality disorder pathway for individuals with primary personality disorder or complex needs including diagnoses of autistic spectrum disorders and other neurocognitive disorders that do not meet criteria for intellectual disability (known in UK health services as learning disability).

At the time of referral to Broadmoor Hospital, the hospital admission panel reviews the referral letter, and two pre-admission assessments completed by Broadmoor staff, a medical assessment by a consultant forensic psychiatrist and a social work assessment by the forensic social worker. Based on the information presented 


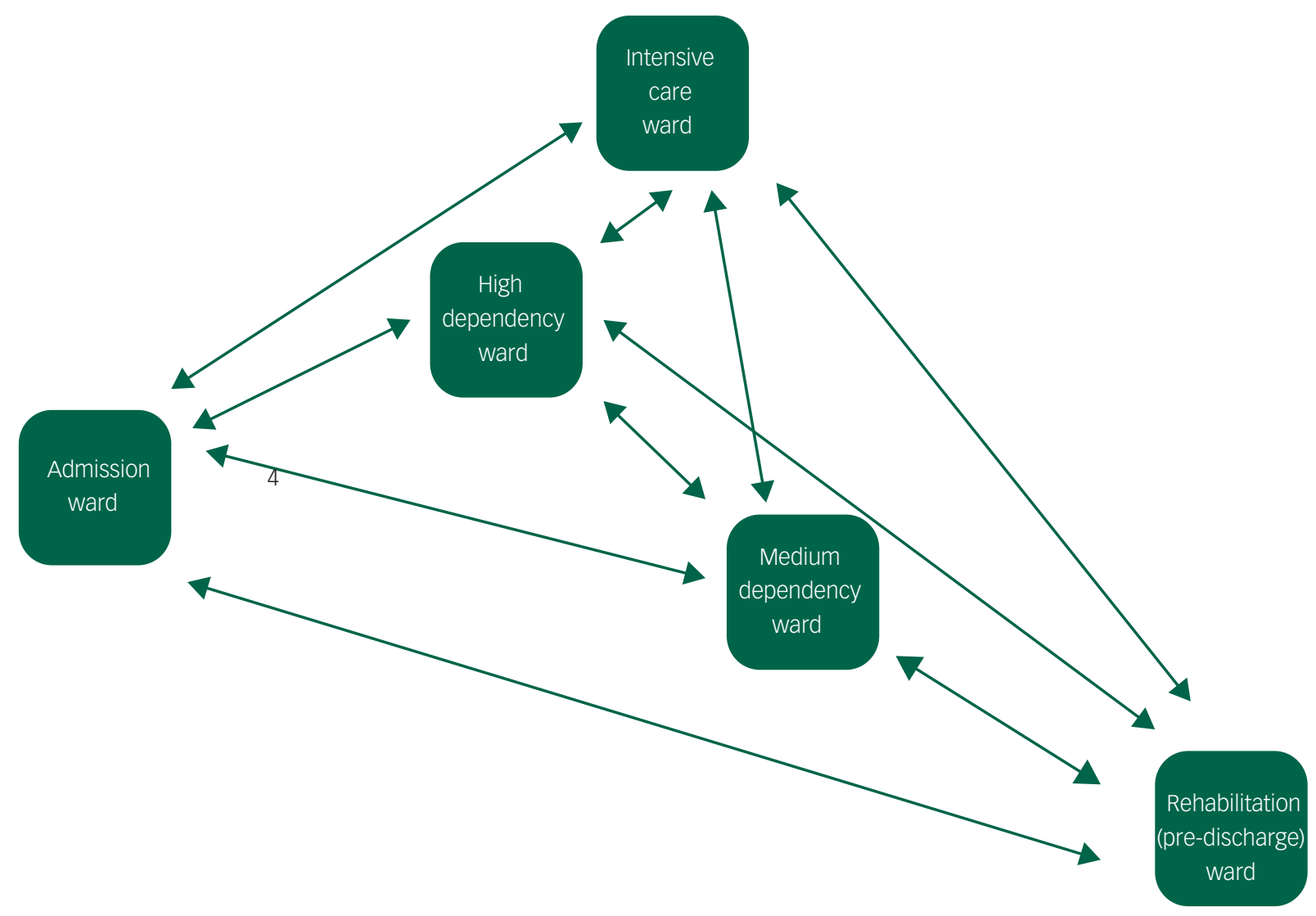

Fig. 1 The care pathway in Broadmoor High Secure Hospital.

The arrows indicate that patients can move between levels of dependency as per their individual needs. The only moves not permitted are moves back onto admission wards once the patient has moved on to a new ward in any direction.

in these three documents the panel decide if the patient requires high secure care and if so whether this should be on the personality disorder or mental illness pathway. There is a high degree of flexibility between pathways in the hospital, patients can move between pathways during the course of their admission if it is considered this might best meet their clinical needs. Within Broadmoor Hospital, high secure care is offered at a number of different levels of therapeutic security, including one intensive care ward as well as admission, high dependency, medium dependency and assertive rehabilitation wards (Fig. 1).

Moves between levels of security within Broadmoor are highly flexible and based on individual patient needs and risk. The only exception to this is that once a patient leaves an admission ward they cannot move back to admissions during their stay. Moves between all other wards are highly flexible and based on the dynamic presentation of the patient.

\section{Participants}

This is an observational study of a cohort of in-patients in Broadmoor High Secure hospital. At the time of commencement of the study, May and June 2016, there were 192 patients in the hospital, of which a complete set of ratings were available for 142 (Fig. 2). All patients were men and over 18 years of age, as Broadmoor Hospital does not admit women or children.

For the 50 patients on whom incomplete data were available, the reasons for this included patients being newly admitted to the hospital and therefore yet to have their first HCR-20-V3 completed or whose HCR-20-V3 was due to be updated, as well as patients for whom there was insufficient detail available to accurately complete a DUNDRUM-3 and DUNDRUM-4 scale (Fig. 2). There were no other exclusion criteria.

Patients included in the study $(n=142)$ were placed on both the mental illness pathway and personality disorder pathways of the hospital. Ratings for patients at all levels of therapeutic security within the high secure hospital settings were included, namely the intensive care unit (Cranfield Ward), high dependency, admission wards, medium dependency and assertive rehabilitation wards.

\section{Variables}

The variables used were scores on the HCR-20-V3, GAF the DUNDRUM-3 programme completion scale and the DUNDRUM-4 recovery scales. Outcomes included placements on the internal care pathway within Broadmoor Hospital, level of ground leave access granted to each individual patient within Broadmoor Hospital and whether or not they were approved for trial leave to the medium secure units (MSUs) by the receiving MSU team and the Ministry of Justice (UK).

\section{Data sources/measurement}

This is a 13-month prospective observational study of a complete cohort of high secure in-patients. All in-patients in Broadmoor Hospital during May and June of 2016 were assessed for therapeutic programme completion and recovery using the DUNDRUM tool by the research team. The Broadmoor multidisciplinary team led by the consultant forensic psychiatrists assessed the overall functioning of each patient under their care using the GAF. 


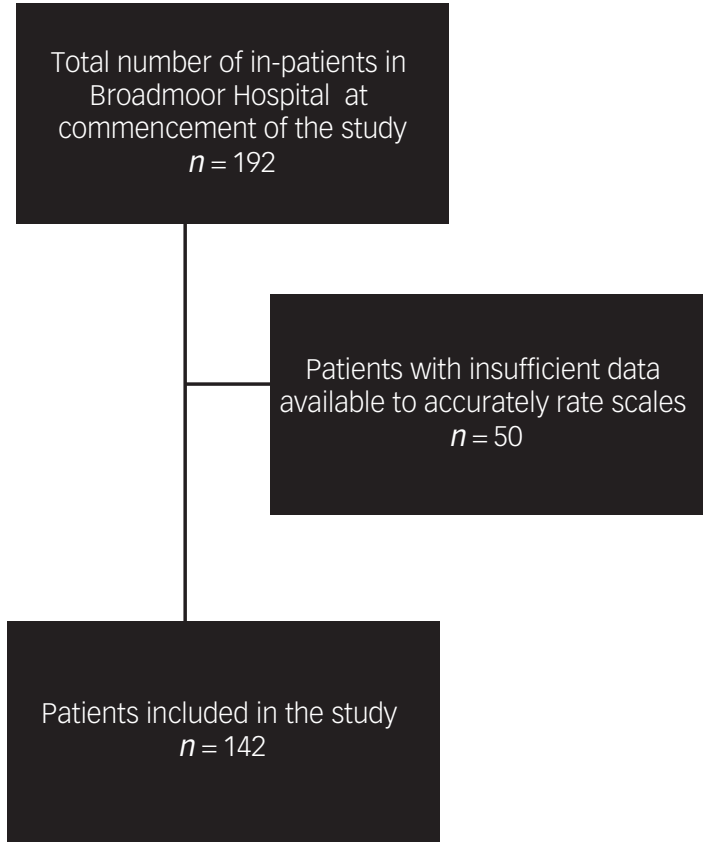

Fig. 2 Study participants.

The HCR-20 violence risk assessment instruments are part of the mandatory outcome measures of the hospital and were collated by the researchers. Each patients' location within the hospital at the time the study commenced (May and June 2016) was noted, and the hospital security team provided information regarding each patient's access to hospital ground leave. The patient group was then observed over the course of 13 months, until June 2017. All moves out of the hospital on trial leave to the MSUs across the south of England were noted.

\section{Bias}

One limitation of this study is that the HCR-20-V3 rating scales were completed by the clinical teams at care programme approach meetings and the GAF scores completed by the Broadmoor responsible clinicians. This might be a potential source of bias. However, only the researchers had access to the scores on the DUNDRUM tools and given that the HCR-20 is a mandatory outcome measure in UK secure forensic hospital settings, it was not deemed appropriate to interfere with treatment as usual in this observational study.

\section{Ethics}

This study was approved as a service evaluation study by the Broadmoor Hospital Audit and Service Evaluation committee. The aim of the study was to ascertain if patients were placed on wards corresponding to ever decreasing levels of therapeutic security within Broadmoor High Secure Hospital according to risk, needs and overall functioning. The study was observational only and did not affect patient care pathways or ground leave decisions in any way. The clinicians attending the security panel for ground leave access did not have access to any of the scores on the tools. The clinicians attending the interward transfer meeting based their views on readiness to move as per the opinion of the receiving consultant and clinical nurse manager, neither of which had seen the outcome of the scores of the tools. This was the usual manner of decisionmaking for moves between units within Broadmoor Hospital.
The decision-making of the two panels, interward transfer panel and security grounds leave panel, was observed only, the research did not in any way affect decision-making regarding the placement, care or treatment of any patient. Likewise, the decision to accept a patient for transfer to their local medium secure unit was made by the receiving MSU team with permission from the Ministry of Justice when needed. These two groups were not aware of the scores on the tools used for the research study. The researchers did not in any way interfere with this decision-making process. Informed consent was therefore not sought from the individual patients placed in the hospital during the period of the study. The authors assert that this work complies with the Helsinki Declaration of 1975, updated in 2008.

\section{Statistical methods}

We investigated the associations between scores of violence risk using HCR-20, scores of therapeutic programme completion using DUNDRUM-3, recovery using DUNDRUM-4 and overall functioning using GAF and (a) placement within the high secure hospital setting and (b) access to grounds leave within the high secure hospital setting. We compared the mean scores on the HCR-20-V3, DUNDRUM-3, DUNDRUM-4 and GAF between those placed on different wards within the high secure hospital care pathway, those offered various levels of ground leave and those approved by MSUs for step down to medium secure hospitals. Mean scores on the tools were compared using ANOVA, a 5\% significance level was adopted for the analysis and SPSS version 21 was used throughout.

\section{Results}

\section{Participants}

Of the 142 adult male high secure in-patients included in the study, 88 (62\%) were from the mental illness pathway, and 54 (38\%) were from the personality disorder pathway. Median length of stay was 4.47 years (mean 6.32 years, s.d. $=5.61$ years). Mean age was 39.5 years (median 38.1 years, s.d. $=10.05$ years). The most common diagnoses were schizophrenia (56.8\%), followed by schizoaffective disorder (11.4\%), dissocial personality disorder (15.3\%) and neurocognitive disorders $(6.8 \%)$. Of note, patients could have more than one diagnosis.

There was no association between diagnosis and length of stay $\left(\chi^{2}=3.377\right.$, d.f. $\left.=7, P=0.848\right)$. The most common Mental Health Act sections were hospital order with restrictions (UK Mental Health Act section 37/41) 47.9\%, prison transfer sections 33.3\%, hybrid order $4.2 \%$ with small numbers of patients detained as unrestricted patients (see Appendix for definitions of terms). Length of stay greater than the mean ( $>5$ years) was associated with hospital order with restrictions $\left(\chi^{2}=40.54\right.$, d.f. $\left.=7, P<0.001\right)$.

\section{Main results}

Placements on the internal high secure hospital care pathway at baseline

The data included patients from both the mental illness and personality disorder pathways of the hospital and also included all levels of therapeutic security within the high security hospital, namely intensive care, high dependency, admissions, medium dependency and assertive rehabilitation wards.

We found that placements on the care pathway within Broadmoor Hospital were closely associated with dynamic measures of risk of violence to others (Table 1). There was no significant association between placements within the high security hospital and historical measures of violence, however, current, future and 


\begin{tabular}{|c|c|c|c|c|c|c|c|c|}
\hline & $\begin{array}{l}\text { Complete } \\
\text { cohort, } \\
\text { mean (s.d.) } \\
(n=142)\end{array}$ & $\begin{array}{c}\text { ICU, mean } \\
(\text { s.d. }) \\
(n=8)\end{array}$ & $\begin{array}{l}\text { HDU, mean } \\
\quad(s . d .) \\
(n=35)\end{array}$ & $\begin{array}{l}\text { Admissions, } \\
\text { mean (s.d.) } \\
(n=29)\end{array}$ & $\begin{array}{c}\text { Medium } \\
\text { dependency, } \\
\text { mean (s.d.) }(n=10)\end{array}$ & $\begin{array}{l}\text { Rehabilitation, } \\
\text { mean (s.d.) } \\
(n=60)\end{array}$ & $\begin{array}{c}\text { ANOVA, } \\
F\end{array}$ & $P$ \\
\hline HCR-Historical scale & $17.0(2.6)$ & $17.0(3.2)$ & $17.6(2.3)$ & $16.8(2.6)$ & $17.3(3.4)$ & $16.8(2.6)$ & 0.596 & 0.666 \\
\hline HCR-Clinical scale & $6.8(2.8)$ & $9.25(1.2)$ & $8.38(2.7)$ & $7.55(1.8)$ & $7.2(1.3)$ & $5.05(2.6)$ & 14.510 & $<0.001$ \\
\hline HCR-Risk scale & $7.0(2.5)$ & $8.38(1.9)$ & $8.62(2.4)$ & 7.40 (1.5) & $6.80(1.5)$ & $5.64(2.3)$ & 11.903 & $<0.001$ \\
\hline $\begin{array}{l}\text { HCR-Dynamic scale (Clinical and } \\
\text { Risk scales combined) }\end{array}$ & $13.8(4.9)$ & $17.63(1.5)$ & $17.00(4.8)$ & $15.00(3.0)$ & $14.0(2.1)$ & $10.69(4.4)$ & 16.324 & $<0.001$ \\
\hline DUNDRUM-3 programme completion & $24.2(2.7)$ & $25.63(1.6)$ & $25.08(2.4)$ & $24.93(2.0)$ & $24.10(2.8)$ & $23.25(2.9)$ & 4.167 & 0.003 \\
\hline DUNDRUM-4 recovery & $23.6(2.3)$ & $25.63(1.7)$ & $24.08(1.4)$ & $23.48(2.7)$ & $23.00(2.0)$ & $23.30(2.4)$ & 2.440 & 0.050 \\
\hline Global Assessment of Functioning & $45.0(20.4)$ & $12.14(7.7)$ & $32.57(16.8)$ & $43.23(17.3)$ & $46.50(12.7)$ & $56.65(16.9)$ & 21.835 & $<0.001$ \\
\hline
\end{tabular}

dynamic scores on HCR-20-V3 were all significantly associated with placement on the internal high secure care pathway, with those whose risks were rated lower being placed on the lower staffed, more open rehabilitation wards in a stepwise manner (Table 1).

Placements on the internal high secure hospital pathway also corresponded to measures of therapeutic programme completion as measured by the DUNDRUM-3 programme completion scale with patients who had successfully completed more therapeutic programmes being placed on lower secure wards, and a similar result was found for the DUNDRUM-4 recovery scale (Table 1).

Measures of overall functioning also clearly corresponded to placement on the high secure hospital care pathway, with the patients whose functioning was poorest being placed on the intensive care unit ward, and mean functioning scores increased in a stepwise manner such that the highest functioning patients were on the rehabilitation wards (Table 1).

\section{Grounds access within the high secure hospital setting at baseline}

In-patients in Broadmoor High Secure Hospital do not have access to structured programmes of community leave, as is the case for all high security forensic hospitals in England. Therefore, we utilised grounds access as a proxy for leave in this study.

Broadmoor High Secure Hospital operates a carefully risk assessed and structured system of supporting in-patients to have access to hospital grounds and off-ward areas. Three levels of grounds access are permitted within the high secure hospital.

(a) Yellow grounds access is the lowest level of access and permits patients to move off ward in groups, supervised by staff, to have access to off-ward gym, café and library and other areas within the high secure hospital. This can be approved by the treating consultant-led multidisciplinary team.

(b) The next level of grounds access, green grounds access, allows patients to independently walk on the large Victorian terrace of the hospital and to visit peers on other rehabilitation wards within the hospital.

(c) The highest level of grounds access is red grounds access. This allows a patient to take a job in the hospital hairdresser, café or shop, thereby allowing them access to risk items or tools.

Green grounds access and red ground access can only be approved by an independent hospital committee, led by the security team. This committee review the patients' clinical notes particularly focusing on engagement and any recent incidents or security issues prior to granting green or red grounds access.

In this study we found that better (lower) scores on the dynamic items of HCR-20-V3 violence risk assessment tool, better (lower) scores on DUNDRUM-3 programme completion and DUNDRUM-4 recovery scales and better (higher) scores on GAF were significantly associated with higher levels of leave within the hospital grounds. We also found that the scores on each of the measures improved in a stepwise manner with the lowest being the group on yellow grounds access, then green grounds access and the best being for those on red grounds access (Table 2).

\section{Trial leave from Broadmoor High Secure Hospital to medium secure hospital settings}

We observed the 142 patient group prospectively over a 13-month period (May 2016 to June 2017) to ascertain which patients would go on to be offered trial leave from the high secure hospital to MSUs.

High secure forensic hospital patients step down to medium secure hospitals once sufficiently stabilised in terms of their mental health and violence risk. For Broadmoor High Secure Hospital, which has a catchment area of London and the South of England, this includes a total of 13 different medium secure hospitals across this area. Decisions regarding readiness to step down are taken over a number of steps. The Broadmoor consultant will refer to the catchment area MSU and the receiving consultant in the MSU will assess the patient and make a recommendation. If step down is approved, this takes place in a process of trial leave for either a 6month or more commonly 1-year period, during which time the patient is placed in the MSU but the Broadmoor consultant retains the role of responsible clinician under the Mental Health Act 1983. In the case of restricted patients, the Ministry of Justice will also need to approve the transfer. If trial leave goes well the patient is formally transferred over at the end of the agreed period. We know from internal hospital audit data that approximately $10 \%$ of trial leaves result in a return to the high secure setting.

During the 13-month follow-up a total of 30 patients were transferred out of Broadmoor to MSUs in England on trial leave. We found that transfer to a medium secure hospital on trial leave was associated with better scores on HCR-20-V3 clinical items, HCR-20-V3 future items, HCR-20-V3 dynamic items, better scores on the DUNDRUM-3 therapeutic programme completion scale and DUNDRUM-4 recovery scale as well as higher overall functioning as measured by GAF (Table 3). It was not associated with HCR-20-Historical or HCR-20-Risk (Future) item scales (Table 3$)$. We noted that a total of three patients (10\%) had unsuccessful trial leaves and needed to return to Broadmoor. It was not possible to analyse the associations with unsuccessful trial leave because of the limited numbers.

\section{Discussion}

We found that placements on the internal high secure hospital pathway corresponded to measures of violence risk, therapeutic 
Table 2 Mean scores on measures of violence risk, therapeutic programme completion, recovery and overall functioning and levels of grounds access at Broadmoor High Secure Hospital ${ }^{a}$

\begin{tabular}{|c|c|c|c|c|c|}
\hline & $\begin{array}{l}\text { Yellow }^{\text {b }} \text { grounds access, } \\
\text { mean (s.d.) }(n=116)\end{array}$ & $\begin{array}{l}\text { Green }^{c} \text { grounds access, } \\
\text { mean (s.d.) }(n=22)\end{array}$ & $\begin{array}{l}\text { Red }^{d} \text { grounds access, } \\
\text { mean (s.d.) }(n=4)\end{array}$ & ANOVA, $F$ & $P$ \\
\hline HCR-Historical scale & $17.079(2.60)$ & $16.955(2.68)$ & $16.000(3.65)$ & 0.331 & 0.718 \\
\hline HCR-Clinical scale & $7.36(2.45)$ & $4.54(2.87)$ & $2.00(1.41)$ & 19.128 & $<0.001$ \\
\hline HCR-Risk scale & $7.38(2.28)$ & $5.45(2.34)$ & $3.75(2.62)$ & 10.523 & $<0.001$ \\
\hline HCR-Dynamic scale (Clinical and Risk scales combined) & $14.75(4.35)$ & $10.00(4.51)$ & $5.75(3.94)$ & 17.789 & $<0.001$ \\
\hline DUNDRUM-3 programme completion & $24.82(2.21)$ & $21.86(3.37)$ & $20.25(2.21)$ & 19.419 & $<0.001$ \\
\hline DUNDRUM-4 recovery & $23.95(2.04)$ & $22.45(2.75)$ & $21.25(3.59)$ & 6.693 & 0.002 \\
\hline Global Assessment of Functioning & $42.11(20.21)$ & $54.48(14.85)$ & $74.00(12.94)$ & 9.757 & $<0.001$ \\
\hline \multicolumn{6}{|c|}{$\begin{array}{l}\text { HCR-20, Historical, Clinical and Risk - } 20 \text {. } \\
\text { a. Both green grounds access and red grounds access require approval by an independent committee chaired by a member of the hospital security team, whereas yellow grounds access } \\
\text { can be granted by the treating multidisciplinary team. } \\
\text { b. Yellow grounds access permits patients to move off their ward in groups supervised by staff to attend off-ward gym and other activities. } \\
\text { c. Green grounds access permits patients to move off their ward to access open walking spaces on the hospital grounds such as the Victorian terrace independently with distant staff } \\
\text { supervision only. } \\
\text { d. Red grounds access is the highest level of grounds access offered to Broadmoor patients. It permits patients to take up a role in the hospital café, shop or hairdresser. }\end{array}$} \\
\hline
\end{tabular}

\begin{tabular}{|c|c|c|c|}
\hline & $\begin{array}{l}\text { Mean score, } \\
\text { mean (s.d.) }\end{array}$ & $\begin{array}{l}\text { ANOVA, } \\
F\end{array}$ & $P$ \\
\hline \multicolumn{4}{|l|}{ HCR-20-V3 Historical scale } \\
\hline Trial leave $(n=30)$ & $17.6(2.2)$ & 1.057 & 0.210 \\
\hline No trial leave $(n=112)$ & $16.9(2.6)$ & & \\
\hline \multicolumn{4}{|l|}{ HCR-20-V3 Historical scale } \\
\hline Trial leave $(n=30)$ & $4.5(2.8)$ & 30.993 & $<0.001$ \\
\hline No trial leave $(n=112)$ & $7.0(2.6)$ & & \\
\hline \multicolumn{4}{|c|}{ HCR-20-V3 Risk (future) scale } \\
\hline Trial leave $(n=30)$ & $5.9(2.2)$ & 22.883 & $<0.001$ \\
\hline No trial leave $(n=112)$ & $7.1(2.4)$ & & \\
\hline \multicolumn{4}{|c|}{$\begin{array}{l}\text { HCR-20-V3 Dynamic (current } \\
\text { and future) scale }\end{array}$} \\
\hline Trial leave $(n=30)$ & $10.5(4.8)$ & 33.199 & $<0.001$ \\
\hline No trial leave $(n=112)$ & $14.1(4.7)$ & & \\
\hline \multicolumn{4}{|l|}{$\begin{array}{l}\text { DUNDRUM-3 programme } \\
\text { completion scale }\end{array}$} \\
\hline Trial leave $(n=30)$ & 22.7 (3.3) & 9.237 & $<0.001$ \\
\hline No trial leave $(n=112)$ & $24.4(2.5)$ & & \\
\hline \multicolumn{4}{|l|}{ DUNDRUM-4 recovery scale } \\
\hline Trial leave $(n=30)$ & $22.0(2.8)$ & 6.863 & 0.001 \\
\hline No trial leave $(n=112)$ & $23.8(2.1)$ & & \\
\hline \multicolumn{4}{|l|}{$\begin{array}{l}\text { Global Assessment of } \\
\text { Functioning }\end{array}$} \\
\hline Trial leave $(n=30)$ & $60.3(16.4)$ & 42.899 & $<0.001$ \\
\hline No trial leave $(n=112)$ & $42.9(20.0)$ & & \\
\hline
\end{tabular}

programme completion, recovery in a broad sense and overall functioning. This is a very important finding and demonstrates that Broadmoor High Secure Hospital is utilising its internal hospital care pathway in a coherent and logical manner, actively implementing the 'least restrictive option' model of care. Access to increased freedoms combined with increased levels of personal responsibility in the area of grounds access within the high secure hospital also corresponded to measures of risk, therapeutic programme completion, recovery and overall functioning. This was a strong finding as access to such grounds access is authorised by an independent security-led committee, rather than the treating clinical team. It is a strength of this methodology, as the clinical decision-makers for the in-patient group were masked to the measures of therapeutic programme completion and recovery, although not to measures of risk.

Approval for transfer to medium secure hospitals on trial leave was also associated with lower current risk of violence, better therapeutic programme completion, better recovery and higher overall functioning. Again, this was a strong finding as the associations between the tools and the independent assessing clinicians from a number of different MSUs were strong. It also demonstrates the effectiveness of the high secure hospital given the significant numbers of patients offered trial leaves and the relatively low rate of unsuccessful trial leaves. We are of the view that a $10 \%$ rate of unsuccessful trial leaves is appropriate. A rate of lower than $10 \%$ would likely indicate that patients could have been moved out of high security in a more timely manner, whereas a higher rate would likely mean that patients were being moved out prior to having achieved the necessary stabilisation and recovery needed to be safely cared for in lower secure settings.

\section{Limitations}

One limitation of this study is that the HCR-20-V3 rating scales were completed by the clinical teams at care programme approach meetings and the GAF scores completed by the Broadmoor responsible clinicians. This might be a potential source of bias. However, the clinical teams were masked to the scores on the DUNDRUM3 programme completion and DUNDRUM- 4 recovery scales. The grounds access committee had no access to the GAF or DUNDRUM scores, nor did the receiving MSU clinicians or the Ministry of Justice.

\section{Interpretations}

Clinically outcomes such as placement on less secure wards within their hospital, increased grounds access and most importantly successful transfer to medium secure hospitals are some of the most important outcomes for patients, their families and supporters, doctors and clinical teams within Broadmoor and other high secure hospital settings. This study clearly demonstrates the practical use of the HCR-20-V3, DUNDRUM-3 programme completion, DUNDRUM-4 recovery scale and GAF as real-world outcome measures in high secure forensic hospital settings.

The dynamic nature of the current and future scales of HCR-20V3, DUNDRUM-3, DUNDRUM-4 and GAF are useful to demonstrate clinically meaningful improvement in this highly vulnerable patient group. The DUNDRUM-3 programme completion and DUNDRUM-4 recovery scales are designed specifically to support clinical decision-making regarding moves to less secure hospital settings and are measured in units of meaningful change, so a mean reduction of one unit across all the items of these two tools should signify readiness to move to a less secure setting.

It is important to note that both the HCR-20-V3 and DUNDRUM tools are SPJ instruments, so although they guide a 
clinician they do not make the final decision, this rests with the treating clinician at all times. The associations between the scores on the DUNDRUM-3 programme completion and DUNDRUM-4 recovery scales and placement within the high secure hospital as well as moves on to medium security demonstrate that clinicians take more than violence risk into account when making such decisions. Important other areas include therapeutic rapport and working alliance, insight, stability of mental state and victim sensitivity and public confidence issues, and clinicians are expected to comment on these for the Ministry of Justice in England when making applications for trial leave from high security. The DUNDRUM tool allows these areas to be systematically rated and assessed, and the HCR-20-V3 allows violence to others to be systematically rated and assessed.

\section{Generalisability}

It is likely that the results found in this study in Broadmoor High Secure Hospital are generalisable to the other high secure hospitals in the UK and also to MSUs internationally. The aim of the HCR$20-\mathrm{V} 3$ and DUNDRUM programme completion and recovery tools is to guide the clinician towards areas of violence risk, therapeutic programme completion and recovery that can be targeted with a combination of medication, individual therapy, group therapies and other psychosocial interventions, therefore supporting the recovery pathway for patients admitted to secure forensic hospital settings. This provides clarity for patients regarding the goals they need to achieve to progress.

The DUNDRUM-3 programme completion tool supports an individualised approach to the care and treatment programmes offered to individual patients. It rates progress in domains rather than dictating particular programmes. For example, patients are rated for successful programme completion in domains such as mental health, offending behaviour, substance misuse or education, occupation and creativity. However, it does not specify the particular programme that must be completed for the patient's progress to be rated, nor does it specify whether or not these programmes should take place in one-to-one or group settings. This is designed specifically to allow an individualised approach to patient care and allow the unique needs and strengths of each patient to be the deciding factor for the best care and treatment approach. It also allows clinicians to think creatively and consider new therapeutic options as they arise. This also ensures that the tool is applicable in different jurisdictions where treatment programmes may take different forms.

We are of the view that regardless of the setting, forensic clinicians take more into account than violence risk alone when moving patients forward on their care pathways - engagement, therapeutic working alliance and recovery in a broad sense are also important.

This study demonstrates the clinical utility of measures of risk, recovery, programme completion and functioning and these tools would therefore be useful to provide routine outcome measurements in high secure forensic hospitals. They also have value in supporting the clinician by offering evidence-based measures of individual patient progress to legal bodies such as mental health tribunals, criminal law review board panels, where violence risk in combination with overall recovery and stability is linked to decision-making. ${ }^{28,37}$

Future research should be undertaken to examine whether or not measures of recovery in a broad sense and therapeutic programme completion, in addition to measures of risk, are linked with longer-term real-world outcomes that are meaningful for high security patients, their families and for the services. These include successful completion of transfer to medium secure forensic hospital settings, recall after unsuccessful transfers to medium secure hospitals as well as successful completion of care with discharge to the community.

Samantha McCullough, BSc (Hons) Psychology, MSC (Forensic Psychology), BPS, Broadmoor Hospital and West London NHS Trust, UK; Carolyn Stanley, MB MRCPsych, North East London Forensic Mental Health Services, Camlet Lodge, Chase Farm Hospital and Barnet, Enfield and Harringey NHS Trust, UK; Helen Smith, BSC (Hons) Psychology, MBPSS, Broadmoor Hospital and West London NHS Trust, UK; Molly Scott, BSC (Hons) Psychology, MSC, MBPSS, Broadmoor Hospital and West London NHS Trust, UK;

Minesh Karia, MBCHB, BMEDSC, Berkshire NHS Foundation Trust, UK;

Benignus Ndubuisi, West London NHS Trust, UK; Callum C. Ross, MB MRCPsych,

Broadmoor Hospital and West London NHS Trust, UK; Rob Bates, MB, MRCPsych,

Broadmoor Hospital and West London NHS Trust, UK; Mary Davoren (D), MB BCh BAO, MD, MRCPsych, Broadmoor Hospital and West London NHS Trust, UK

Correspondence: Mary Davoren. Email: davorem@tcd.ie

First received 16 Apr 2020, final revision 20 Jun 2020, accepted 22 Jun 2020

\section{Acknowledgements}

The authors would like to acknowledge the medical records department at Broadmoor Hospital for their support in completing this study.

\section{Data availability}

As a result of the uniquely sensitive nature of this data-set, access to data will only be provided on a strict case-by-case basis.

\section{Author contributions}

M.D. designed the study and formulated the research question. S.MCC., C.S., H.S., M.S., M.K. and B.N. rated the DUNDRUM tool and collated the GAF and HCR-20 results. M.D. analysed the data. All contributed to the authorship of the paper.

\section{Declaration of interest}

None.

ICMJE forms are in the supplementary material, available online at https://doi.org/10.1192/ bjo.2020.61.

\section{Appendix}

Hospital order with restrictions under section 37/41 of the Mental Health Act (England) is a hospital disposal with the restriction that decisions regarding discharge must be taken by a mental health tribunal and approved by the Ministry of Justice. Decisions regarding community leave or moves between hospitals at different levels of security must also be approved by the Ministry of Justice.

Prison transfer sections of the Mental Health Act (England) allow for the involuntary admission of remand or sentenced prisoners to forensic hospital settings. This may or may not have restrictions attached.

A hybrid order (section 45(a)) of the Mental Health Act (England) provides that at the time of conviction a patient can be directed to hospital and once it is deemed that they no longer require in-patient treatment they can be transferred back to prison to complete their sentence. Decisions regarding release are taken by the parole board, not the mental health tribunal system.

Unrestricted sections of the Mental Health Act (England) comprise hospital orders without Ministry of Justice restrictions.

\section{References}

1 Kennedy HG. Therapeutic uses of security: mapping forensic mental health services by stratifying risk. Adv Psychiatr Treat 2002; 8: 433-43.

2 Rutherford M, Duggan S. Forensic Mental Health Services: Facts and Figures on Current Provision. Sainsbury Centre for Mental Health, 2007.

3 Coid J, Kahtan N, Cook A, Gault S, Jarman B. Predicting admission rates to secure forensic psychiatry services. Psychol Med 2001; 31: 531-9. 
4 Edge D, Walker T, Meacock R, Wilson H, McNair L, Shaw J, et al. Secure pathways for women in the UK: lessons from the women's enhanced medium secure services (WEMSS) pilots. J Forens Psychiatry Psychol 2017; 28: 206-25.

5 Duke L H, Furtado V, Guo B, Vollm BA. Long-stay in forensic-psychiatric care in the UK. Soc Psychiatry Psychiatr Epidemiol 2018; 53: 313-21.

6 Williams HK, Senanayke M, Ross CC, Bates R, Davoren M. Security needs among patients referred for high secure care in Broadmoor Hospital England. BJPsych Open 2020; 6: e55.

7 Wilson S, James D, Forrester A. The medium-secure project and criminal justice mental health. Lancet 2011; 378: 110-1.

8 Pillay SM, Oliver B, Butler L, Kennedy HG. Risk stratification and the care pathway. Ir J Psychol Med 2008; 25: 123-7.

9 Jeandarme I, Saloppé X, Habets $P$, Pham TH. Not guilty by reason of insanity: clinical and judicial profile of medium and high security patients in Belgium. $J$ Forensic Psychiatry Psychol 2019; 30: 286-300.

10 Adams J, Thomas SD, Mackinnon T, Eggleton D. The risks, needs and stages of recovery of a complete forensic patient cohort in an Australian state. BMC Psychiatry 2018; 18: 35.

11 World Health Organization. Mental Health Care Law: Ten Basic Principles: With Annotations Suggesting Selected Actions to Promote their Implementation. World Health Organization, 1996.

12 Department of Health. Mental Health Act 1983: Code Of Practice 3rd ed: 98-338. TSO, 2015.

13 Douglas KS, Webster CD, Hart SD, Belfrage H. Mental Health Law and Policy Institute, Forensic Psychiatric Services Commission of British Columbia. HCR20v3: Assessing Risk for Violence: User Guide (3rd edn). Mental Health, Law, and Policy Institute, Simon Fraser University; 2013.

14 Cartwright JK, Desmarais SL, Hazel J, Griffith T, Azizian A. Predictive validity of HCR-20, START, and static-99R assessments in predicting institutional aggression among sexual offenders. Law Hum Behav 2018; 42: 13-25.

15 O'Shea LE, Dickens GL. The HCR-20 as a measure of reliable and clinically significant change in violence risk among secure psychiatric inpatients. Compr Psychiatry 2015; 62: 132-40.

16 Shepherd SM, Campbell RE, Ogloff JRP. The Utility of the HCR-20 in an Australian sample of forensic psychiatric patients. Psychiatr Psychol Law 2018; 25: 273-82.

17 O'Shea LE, Thaker DK, Picchioni MM, Mason FL, Knight C, Dickens GL. Predictive validity of the HCR-20 for violent and non-violent sexual behaviour in a secure mental health service. Crim Behav Ment Health 2016; 26: 366-79.

18 Richter MS. O'Reilly K, O'Sullivan D, O'Flynn P, Corvin A, Donohoe G, et al Prospective observational cohort study of 'treatment as usual' over four years for patients with schizophrenia in a national forensic hospital. BMC Psychiatry 2018; 18: 289

19 Webster CD. Mental Health Law and Policy Institute. HCR-20: Assessing Risk for Violence (2nd edn). Mental Health, Law, and Policy Institute, Simon Fraser University in cooperation with the British Columbia Forensic Psychiatric Services Commission; 1997.

20 Kennedy H, O'Neill C, Flynn G, Gill P, Davoren M. The DUNDRUM Toolkit Draft V1. 0.30. Trinity College Dublin, 2016

21 Flynn G, O'Neill C, McInerney C, Kennedy HG. The DUNDRUM-1 structured professional judgment for triage to appropriate levels of therapeutic security: retrospective-cohort validation study. BMC Psychiatry 2011; 11: 43.

22 Flynn G, O'Neill C, Kennedy HG. DUNDRUM-2: prospective validation of a structured professional judgment instrument assessing priority for admission from the waiting list for a forensic mental health hospital. BMC Res Notes 2011; 4: 230.
23 Freestone M, Bull D, Brown R, Boast N, Blazey F, Gilluley P. Triage, decisionmaking and follow-up of patients referred to a UK forensic service: validation of the DUNDRUM toolkit. BMC Psychiatry 2015; 15: 239

24 Habets $P$, Jeandarme I, Kennedy HG. Applicability of the DUNDRUM-1 in a forensic Belgium setting. J Forensic Pract 2019; 21: 85-94.

25 Jeandarme I, Habets P. Determining security need in forensic psychiatric patients: HoNOS-Secure and DUNDRUM-1. Tijdschr Psychiatr 2019; 61: 455-63.

26 Jeandarme I, Habets $P$, Kennedy $H$. Structured versus unstructured judgment: DUNDRUM-1 compared to court decisions. Int J Law Psychiatry 2019; 64: 20510.

27 Jones RM, Patel K, Simpson AIF. Assessment of need for inpatient treatment for mental disorder among female prisoners: a cross-sectional study of provincially detained women in Ontario. BMC Psychiatry 2019; 19: 98.

28 Davoren M, Abidin Z, Naughton L, Gibbons O, Nulty A, Wright B, et al. Prospective study of factors influencing conditional discharge from a forensic hospital: the DUNDRUM-3 programme completion and DUNDRUM-4 recovery structured professional judgement instruments and risk. BMC Psychiatry 2013; 13: 185.

29 Davoren M, O'Dwyer S, Abidin Z, Naughton L, Gibbons O, Doyle E, et al Prospective in-patient cohort study of moves between levels of therapeutic security: the DUNDRUM-1 triage security, DUNDRUM-3 programme completion and DUNDRUM-4 recovery scales and the HCR-20. BMC Psychiatry 2012; 12: 80 .

30 O'Dwyer S, Davoren M, Abidin Z, Doyle E, McDonnell K, Kennedy HG. The DUNDRUM Quartet: validation of structured professional judgement instruments DUNDRUM-3 assessment of programme completion and DUNDRUM-4 assessment of recovery in forensic mental health services. BMC Res Notes 2011; 4: 229.

31 Abidin Z, Davoren M, Naughton L, Gibbons O, Nulty A, Kennedy HG. Susceptibility (risk and protective) factors for in-patient violence and selfharm: prospective study of structured professional judgement instruments START and SAPROF, DUNDRUM-3 and DUNDRUM-4 in forensic mental health services. BMC Psychiatry 2013; 13: 197.

32 Wharewera-Mika J, Cooper E, Wiki N, Prentice K, Field T, Cavney J, et al. The appropriateness of DUNDRUM-3 and DUNDRUM-4 for Maori in forensic mental health services in New Zealand: participatory action research. BMC Psychiatry 2020; 20: 61

33 Davoren M, Hennessy S, Conway C, Marrinan S, Gill P, Kennedy HG. Recovery and concordance in a secure forensic psychiatry hospital - the self rated DUNDRUM-3 programme completion and DUNDRUM-4 recovery scales. BMC Psychiatry 2015; 15: 61

34 Shinkfield G, Ogloff J. Use and interpretation of routine outcome measures in forensic mental health. Int J Ment Health Nurs 2015; 24: 11-8.

35 Keulen-de Vos M, Schepers K. Needs Assessment in Forensic Patients: A Review of Instrument Suites. Routledge, 2016.

36 AmericanPsychiatricAssociation. Diagnostic and Statistical Manual of Mental Disorders: DSM-IV. APA, 1994.

37 Crocker AG, Nicholls TL, Charette $Y$, Seto MC. Dynamic and static factors associated with discharge dispositions: the national trajectory project of individuals found not criminally responsible on account of mental disorder (NCRMD) in Canada. Behav Sci Law 2014: 32: 577-95.
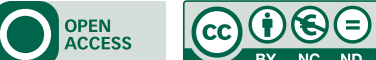